\title{
A Comparative Analysis of Secrecy Rates of Wireless Two-Way Relay Systems
}

\author{
Chih-Hua Chang*, Ronald Y. Chang ${ }^{\dagger}$, and Yu-Chih Huang ${ }^{\ddagger}$ \\ ${ }^{*}$ School of Electrical and Computer Engineering, Purdue University, USA \\ ${ }^{\dagger}$ Research Center for Information Technology Innovation, Academia Sinica, Taiwan \\ $\ddagger$ Department of Communication Engineering, National Taipei University, Taiwan \\ Email: chang377@purdue.edu,rchang@ citi.sinica.edu.tw, ychuang@mail.ntpu.edu.tw
}

\begin{abstract}
This paper studies the information-theoretic secrecy rates of wireless two-way relay systems where two users wish to exchange information through a single relay with an eavesdropper observing all communications. We formulate and compare the achievable secrecy rates of the system that employs one of the three common relay protocols: conventional decode-and-forward (DF), DF with network coding (NC), and compute-and-forward (CF) based on physical-layer network coding (PNC). We show that CF based on PNC achieves the highest secrecy rate at high signal-to-noise ratio (SNR), while, interestingly, the other two protocols have mixed performance depending on the power allocation scheme and network topology. Our study offers insights into designing wireless two-way relay protocols from a secrecy perspective.
\end{abstract}

Index Terms-Two-way relaying, information-theoretic security, physical-layer security, network coding, physical-layer network coding.

\section{INTRODUCTION}

Physical layer security [1] introduces the concept of establishing secure wireless communications through the inherent randomness and location-specific properties of the wireless channel in the physical layer rather than the conventional cryptographic methods in the application layer. The fundamental issues of secure channel capacity for physical layer security have been studied since the pioneer work by Shannon [2]. Wyner [3] showed that perfect secrecy is achievable using physical layer techniques and derived the secrecy capacity for a wiretap channel, which was generalized by Csiszár and Körner [4]. Secrecy capacity of various channels has since been derived, such as Gaussian channels [5], multiple-access channels [6], and relay channels [7], [8].

A specific relay channel, i.e., the two-way relay channel, where two users without direct communication exchange information through a single relay, represents a wide array of applications (e.g., two users communicate through an access point or a satellite). Secure communication in the two-way relay network was studied with an untrusted relay [9], [10] and a trusted relay with external eavesdroppers [11]-[13]. The secrecy capacity region of Gaussian two-way relay wiretap channels was investigated in [11]. The secrecy sum rates of

This work was supported in part by the Ministry of Science and Technology, Taiwan, under Grants MOST 103-2221-E-001-027 and MOST 104-2218-E305-001-MY2. two-way relay networks were studied with single-antenna [12] and multi-antenna nodes [13].

In this work, we consider a Gaussian two-way relay channel with a trusted relay and an observing eavesdropper. We formulate and compare the achievable secrecy rates when one of the three common relay protocols is employed: conventional DF (four-phase), DF with NC (three-phase) [14], and CF based on PNC (two-phase) [15], [16]. Previous comparisons of these protocols (e.g., [17], [18]) have focused on the throughput performance without considering an eavesdropper in presence. The motivation of this work is to conduct a similar comparative study yet from a previously unexamined perspective of achievable secrecy rates. Note that while current research efforts focus on the two-phase scheme, the fourphase scheme is still widely used in commercially available routers, and the three-phase scheme avoids the commonly known challenges such as simultaneous transmissions from the two users as in the two-phase scheme.

Our main results are:

1) We show that the two-phase scheme achieves the highest secrecy rates at high SNR.

2) We show that, unlike the Gaussian two-way relay channel without an eavesdropper, three-phase and fourphase schemes achieve mixed secrecy-rate performance depending on the power allocation and network topology (relative locations of nodes). The crossing points are examined in terms of network topologies based on the indifference curve of achievable secrecy rates.

The outline of this paper is as follows. Sec. II describes the system model. Sec. III formulates the achievable secrecy rates and Sec. IV presents the comparative study. Sec. V presents numerical results and discussion. Finally, Sec. VI concludes this work.

\section{SySTEM MODEL}

We consider a wireless two-way relay system with four arbitrarily located nodes: user $A$, user $B$, relay $R$, and eavesdropper $E$. User $A$ and user $B$ have no direct communication link and intend to exchange their independent messages $\left(W_{A}\right.$ and $W_{B}$, respectively) through a single relay $R$. A passive eavesdropper $E$ can overhear signals from nodes $A, B$, and $R$, and intends to access $W_{A}$ and $W_{B}$. The messages $W_{A}$ and 
(a)

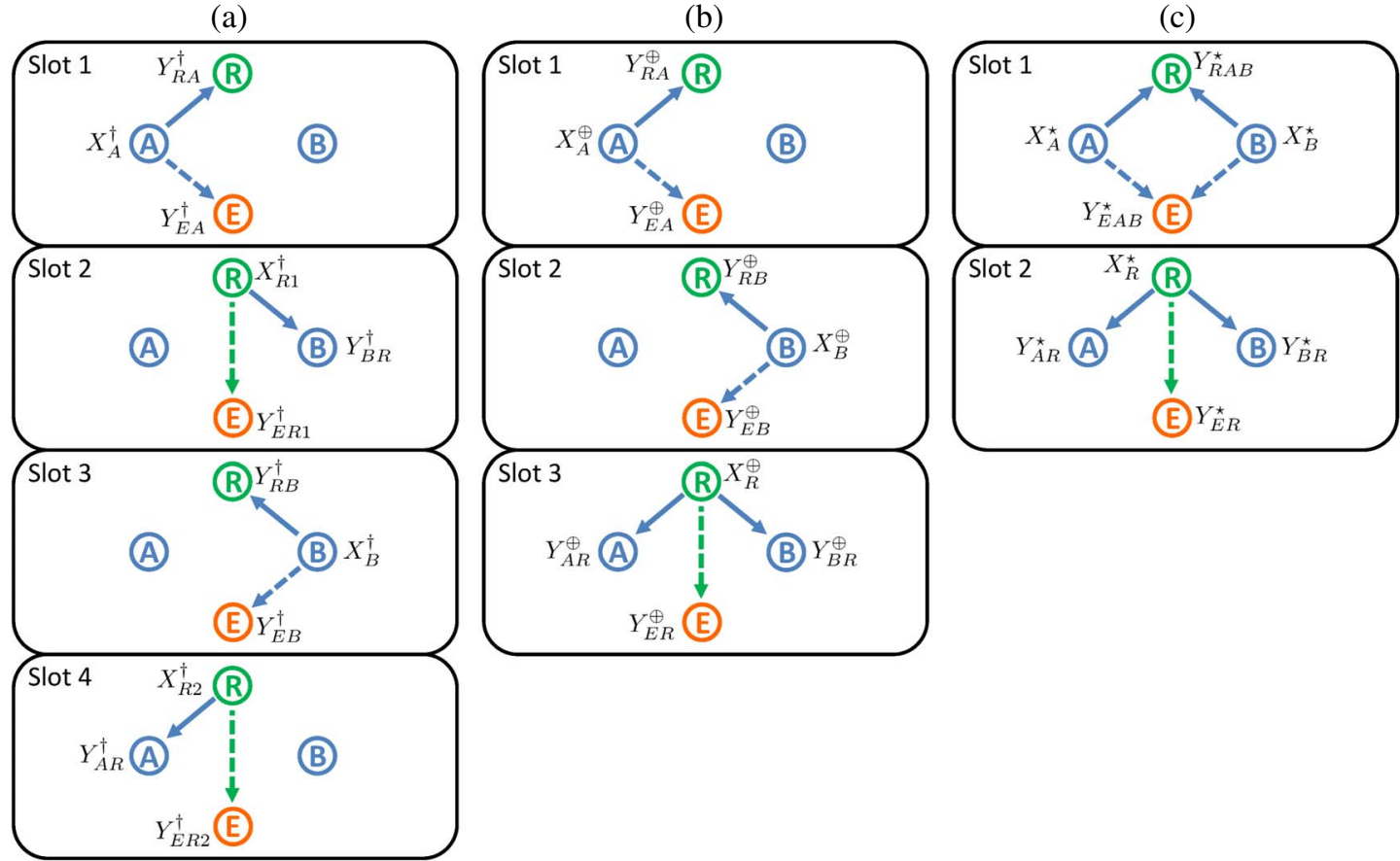

Fig. 1. The procedures of (a) conventional DF, (b) DF with NC, and (c) CF based on PNC, in a wireless two-way relay system where two users $A$ and $B$ exchange information through a single relay $R$ while a passive eavesdropper $E$ observes all communications.

$W_{B}$ are encoded into length- $n$ codewords $X_{A}$ and $X_{B}$, respectively, for transmission. Three relay protocols are considered and compared. The procedures of a) conventional DF, b) DF with $\mathrm{NC}$, and c) $\mathrm{CF}$ based on PNC are illustrated in Fig. 1, where $X(i) \rightarrow Y$ represents that node $i$ transmits signal $X$ to node $j$ and node $j$ receives signal $Y$, and superscripts $\dagger, \oplus$, $\star$ denote message passing in a)-c), respectively. The transmit powers are defined as $P_{i}^{k}=\frac{1}{n} \mathbb{E}\left[\left(X_{i}^{k}\right)^{2}\right], k \in\{\dagger, \oplus, \star\}, i \in$ $\{A, B, R, R 1, R 2\}$. The channel from node $i$ to node $j$ is an additive white Gaussian noise (AWGN) channel with channel gain $h_{i j}$ and noise power $\sigma_{j}^{2}$ at the receiving node $j$, for $i, j \in\{A, B, R, E\}$.

\section{AChievable Secrecy Rate Formulation}

Protocol 1: Conventional DF: Given independent signals $X_{A}^{\dagger}$ and $X_{B}^{\dagger}$ and no direct link between $A$ and $B$, we have [7]

$$
\begin{aligned}
& I\left(X_{A}^{\dagger}, Y_{B R}^{\dagger}\right)=\min \left\{\frac{1}{4} C\left(\gamma_{A R} P_{A}^{\dagger}\right), \frac{1}{4} C\left(\gamma_{R B} P_{R 1}^{\dagger}\right)\right\} \\
& I\left(X_{B}^{\dagger}, Y_{A R}^{\dagger}\right)=\min \left\{\frac{1}{4} C\left(\gamma_{B R} P_{B}^{\dagger}\right), \frac{1}{4} C\left(\gamma_{R A} P_{R 2}^{\dagger}\right)\right\}
\end{aligned}
$$

where $\gamma_{i j} \triangleq\left|h_{i j}\right|^{2} / \sigma_{j}^{2}$ and $C(x) \triangleq \log (1+x)$ for AWGN channels. The factor of $1 / 4$ reflects the fact that Protocol 1 requires four time slots (or phases) to complete transmission. On the other hand, denoting the signal received at the eaves- dropper as $\mathbf{Y}_{E}^{\dagger}=\left\{Y_{E A}^{\dagger}, Y_{E R 1}^{\dagger}, Y_{E B}^{\dagger}, Y_{E R 2}^{\dagger}\right\}$, we have [7]

$I\left(X_{A}^{\dagger}, \mathbf{Y}_{E}^{\dagger}\right)=I\left(X_{A}^{\dagger}, Y_{E A}^{\dagger}, Y_{E R 1}^{\dagger}\right)=\frac{1}{4} C\left(\gamma_{A E} P_{A}^{\dagger}+\gamma_{R E} P_{R 1}^{\dagger}\right)$

$I\left(X_{B}^{\dagger}, \mathbf{Y}_{E}^{\dagger}\right)=I\left(X_{B}^{\dagger}, Y_{E B}^{\dagger}, Y_{E R 2}^{\dagger}\right)=\frac{1}{4} C\left(\gamma_{B E} P_{B}^{\dagger}+\gamma_{R E} P_{R 2}^{\dagger}\right)$

where we have used the fact that $X_{B}^{\dagger}$ (or $X_{A}^{\dagger}$ ) is independent of $\left\{Y_{E A}^{\dagger}, Y_{E R 1}^{\dagger}\right\}$ (or $\left\{Y_{E B}^{\dagger}, Y_{E R 2}^{\dagger}\right\}$ ). Using Gaussian inputs and stochastic encoders with full channel state information (CSI) of all nodes at each transmitter, the achievable secrecy rate for Protocol 1 is given by [13]

$$
\begin{aligned}
R_{s}^{\dagger} & =\left[\sum_{i, j \in\{A, B\}, i \neq j}\left(\frac{1}{2} I\left(X_{i}^{\dagger}, Y_{j R}^{\dagger}\right)-I\left(X_{i}^{\dagger}, \mathbf{Y}_{E}^{\dagger}\right)\right)\right]^{+} \\
& =\left[R_{M}^{\dagger}-R_{E}^{\dagger}\right]^{+}
\end{aligned}
$$

where $R_{M}^{\dagger} \triangleq \frac{1}{2}\left(I\left(X_{A}^{\dagger}, Y_{B R}^{\dagger}\right)+I\left(X_{B}^{\dagger}, Y_{A R}^{\dagger}\right)\right), R_{E}^{\dagger} \triangleq$ $I\left(X_{A}^{\dagger}, \mathbf{Y}_{E}^{\dagger}\right)+I\left(X_{B}^{\dagger}, \mathbf{Y}_{E}^{\dagger}\right)$, and $[a]^{+}=\max (a, 0)$.

Protocol 2: DF with NC: Protocol 2 requires three time slots (or phases) to complete transmission. Thus, we have

$$
\begin{aligned}
& I\left(X_{A}^{\oplus}, Y_{B R}^{\oplus}\right)=\min \left\{\frac{1}{3} C\left(\gamma_{A R} P_{A}^{\oplus}\right), \frac{1}{3} C\left(\gamma_{R B} P_{R}^{\oplus}\right)\right\} \\
& I\left(X_{B}^{\oplus}, Y_{A R}^{\oplus}\right)=\min \left\{\frac{1}{3} C\left(\gamma_{B R} P_{B}^{\oplus}\right), \frac{1}{3} C\left(\gamma_{R A} P_{R}^{\oplus}\right)\right\} .
\end{aligned}
$$

If the eavesdropper observing the signal $\mathbf{Y}_{E}^{\oplus}=$ $\left\{Y_{E A}^{\oplus}, Y_{E B}^{\oplus}, Y_{E R}^{\oplus}\right\}$ adopts maximal ratio combining (MRC), 
we have [19]

$$
\begin{aligned}
I\left(X_{A}^{\oplus}, \mathbf{Y}_{E}^{\oplus}\right) & =\frac{1}{3} C\left(\gamma_{A E} P_{A}^{\oplus}+\gamma_{R E} P_{R}^{\oplus}\right) \\
I\left(X_{B}^{\oplus}, \mathbf{Y}_{E}^{\oplus}\right) & =\frac{1}{3} C\left(\gamma_{B E} P_{B}^{\oplus}+\gamma_{R E} P_{R}^{\oplus}\right) .
\end{aligned}
$$

Similar to Protocol 1, the achievable secrecy rate for Protocol 2 is given by

$$
R_{s}^{\oplus}=\left[R_{M}^{\oplus}-R_{E}^{\oplus}\right]^{+}
$$

where $R_{M}^{\oplus} \triangleq \frac{1}{2}\left(I\left(X_{A}^{\oplus}, Y_{B R}^{\dagger}\right)+I\left(X_{B}^{\oplus}, Y_{A R}^{\oplus}\right)\right)$ and $R_{E}^{\oplus} \triangleq$ $I\left(X_{A}^{\oplus}, \mathbf{Y}_{E}^{\oplus}\right)+I\left(X_{B}^{\oplus}, \mathbf{Y}_{E}^{\oplus}\right)$.

Protocol 3: CF Based on PNC: Protocol 3 requires two time slots (or phases) to complete transmission. Unlike Protocols 1 and 2, the current best known achievable rate of the two-way relay channel with Protocol 3 is not derived using Gaussian inputs but through a lattice coding strategy [16], [18]. Thus, (5) does not apply to Protocol 3. We instead refer to the following theorem, which is based on [11, Theorem 1] with no jamming at both the users and the relay (i.e., $\alpha_{1}=\alpha_{2}=\beta=1$ in [11]) and no open message.

Theorem 1. The secrecy rate $R_{s}^{\star}$ given below is achievable:

$$
R_{s}^{\star} \leq \min \left\{R_{u}^{s}, R_{d}^{s}\right\}
$$

where

$$
\begin{aligned}
& R_{u}^{s} \leq \frac{1}{2} \log \left(\frac{1}{2}+\frac{P^{s}}{\sigma_{R}^{2}}\right) \\
& R_{d}^{s} \leq \frac{1}{2} \min \left\{\log \left(1+\frac{\left|h_{R A}\right|^{2} P_{R}^{\star}}{\sigma_{A}^{2}}\right), \log \left(1+\frac{\left|h_{R B}\right|^{2} P_{R}^{\star}}{\sigma_{B}^{2}}\right)\right\}
\end{aligned}
$$

and $P^{s}$ is the power allocated for the lattice code such that

$$
P^{s} \leq \frac{\left|h_{A R}\right|^{2} P_{A}^{\star}+\left|h_{B R}\right|^{2} P_{B}^{\star}-\left(\gamma_{A E} P_{A}^{\star}+\gamma_{B E} P_{B}^{\star}\right) \sigma_{R}^{2}}{2\left(1+\gamma_{A E} P_{A}^{\star}+\gamma_{B E} P_{B}^{\star}\right)} .
$$

From Theorem 1, substituting (14) into (12) yields

$$
\begin{aligned}
& R_{u}^{s} \leq \frac{1}{2} \log \left(\frac{1}{2}+\frac{\gamma_{A R} P_{A}^{\star}+\gamma_{B R} P_{B}^{\star}-\gamma_{A E} P_{A}^{\star}-\gamma_{B E} P_{B}^{\star}}{2\left(1+\gamma_{A E} P_{A}^{\star}+\gamma_{B E} P_{B}^{\star}\right)}\right) \\
& =\frac{1}{2} \log \left(\frac{1}{2}+\frac{\gamma_{A R} P_{A}^{\star}+\gamma_{B R} P_{B}^{\star}}{2}\right)-\frac{1}{2} C\left(\gamma_{A E} P_{A}^{\star}+\gamma_{B E} P_{B}^{\star}\right) \\
& =R_{T}^{\star}-R_{E}^{\star}
\end{aligned}
$$

where $R_{T}^{\star} \triangleq \frac{1}{2} \log \left(\frac{1}{2}+\frac{\gamma_{A R} P_{A}^{\star}+\gamma_{B R} P_{B}^{\star}}{2}\right)$ and $R_{E}^{\star} \triangleq$ $\frac{1}{2} C\left(\gamma_{A E} P_{A}^{\star}+\gamma_{B E} P_{B}^{\star}\right)$. Combining (11), (13), and (15) leads to the achievable secrecy rate for Protocol 3 given by

$$
R_{s}^{\star}=\min \left\{\left[R_{T}^{\star}-R_{E}^{\star}\right]^{+}, \frac{1}{2} C\left(\gamma_{R A} P_{R}^{\star}\right), \frac{1}{2} C\left(\gamma_{R B} P_{R}^{\star}\right)\right\} .
$$

\section{A Comparative Study}

We perform a comparative analysis of $R_{s}^{\dagger}, R_{s}^{\oplus}$, and $R_{s}^{\star}$ with a common total power constraint $P_{t}$, i.e., $\sum_{p \in \mathcal{P}^{k}} p \leq$ $P_{t}$ for $k \in\{\dagger, \oplus, \star\}$, where $\mathcal{P}^{\dagger}=\left\{P_{A}^{\dagger}, P_{B}^{\dagger}, P_{R 1}^{\dagger}, P_{R 2}^{\dagger}\right\}$, $\mathcal{P}^{\oplus}=\left\{P_{A}^{\oplus}, P_{B}^{\oplus}, P_{R}^{\oplus}\right\}$, and $\mathcal{P}^{\star}=\left\{P_{A}^{\star}, P_{B}^{\star}, P_{R}^{\star}\right\}$. We consider the case where the two users have equal distances to the relay, i.e., $\gamma_{M} \triangleq \gamma_{A R}=\gamma_{R A}=\gamma_{B R}=\gamma_{R B}$. We examine equal power allocation (where power equalization at the relay follows an equal maximum power criterion) and optimal power allocation (which achieves maximum secrecy rates).

\section{A. Equal Power Allocation}

Theorem 2. With equal power allocation satisfying $P_{A}^{\dagger}=$ $P_{A}^{\oplus}=P_{A}^{\star}, P_{B}^{\dagger}=P_{B}^{\oplus}=P_{B}^{\star}$, and $\max \left\{P_{R 1}^{\dagger}, P_{R 2}^{\dagger}\right\}=P_{R}^{\oplus}=$ $P_{R}^{\star}$, we have $R_{s}^{\star} \geq \frac{3}{2} R_{s}^{\oplus}$ and $R_{s}^{\star} \geq 2 R_{s}^{\dagger}$ at high $S N R$, i.e., $\gamma_{M}\left(P_{A}^{\star}+P_{B}^{\star}\right) \gg 1$. If the power allocation further satisfies $P_{R 1}^{\dagger}=P_{R 2}^{\dagger}=P_{R}^{\oplus}=P_{R}^{\star}$, we have $R_{s}^{\star} \geq \frac{3}{2} R_{s}^{\oplus} \geq 2 R_{s}^{\dagger}$. leads to

Proof: Substituting $\gamma_{A R}=\gamma_{R A}=\gamma_{B R}=\gamma_{R B}=\gamma_{M}$ in (16)

$$
\begin{aligned}
R_{s}^{\star} & =\min \left\{\left[R_{T}^{\star}-R_{E}^{\star}\right]^{+}, \frac{1}{2} C\left(\gamma_{M} P_{R}^{\star}\right)\right\} \\
& \stackrel{(a)}{=}\left[\min \left\{R_{T}^{\star}-R_{E}^{\star}, \frac{1}{2} C\left(\gamma_{M} P_{R}^{\star}\right)\right\}\right]^{+} \\
& \geq\left[\min \left\{R_{T}^{\star}, \frac{1}{2} C\left(\gamma_{M} P_{R}^{\star}\right)\right\}-R_{E}^{\star}\right]^{+} \\
& =\left[R_{M}^{\star}-R_{E}^{\star}\right]^{+}
\end{aligned}
$$

where $(a)$ holds since $C\left(\gamma_{M} P_{R}^{\star}\right) \geq 0$, and $R_{M}^{\star} \triangleq$ $\min \left\{R_{T}^{\star}, \frac{1}{2} C\left(\gamma_{M} P_{R}^{\star}\right)\right\}$. Since $\gamma_{M}\left(P_{A}^{\star}+P_{B}^{\star}\right) \gg 1$, we have $R_{T}^{\star} \simeq \frac{1}{2} \log \left(1+\frac{\gamma_{A R} P_{A}^{\star}+\gamma_{B R} P_{B}^{\star}}{2}\right)$ and consequently

$$
\begin{aligned}
2 R_{T}^{\star} & \simeq \log \left(\frac{2+\gamma_{M} P_{A}^{\star}+\gamma_{M} P_{B}^{\star}}{2}\right) \\
& \geq \frac{1}{2} \log \left(1+\gamma_{M} P_{A}^{\star}\right)+\frac{1}{2} \log \left(1+\gamma_{M} P_{B}^{\star}\right) \\
& =\frac{1}{2} C\left(\gamma_{M} P_{A}^{\star}\right)+\frac{1}{2} C\left(\gamma_{M} P_{B}^{\star}\right)
\end{aligned}
$$

where the inequality follows from the Jensen's inequality. With $P_{A}^{\dagger}=P_{A}^{\oplus}=P_{A}^{\star}, P_{B}^{\dagger}=P_{B}^{\oplus}=P_{B}^{\star}$, and $\max \left\{P_{R 1}^{\dagger}, P_{R 2}^{\dagger}\right\}=$ $P_{R}^{\oplus}=P_{R}^{\star}$, we can derive

$$
\begin{aligned}
2 R_{M}^{\star}= & \min \left\{2 R_{T}^{\star}, C\left(\gamma_{M} P_{R}^{\star}\right)\right\} \\
\geq & \min \left\{\frac{1}{2} C\left(\gamma_{M} P_{A}^{\oplus}\right)+\frac{1}{2} C\left(\gamma_{M} P_{B}^{\oplus}\right), C\left(\gamma_{M} P_{R}^{\oplus}\right)\right\} \\
\geq & \min \left\{\frac{1}{2} C\left(\gamma_{M} P_{A}^{\oplus}\right), \frac{1}{2} C\left(\gamma_{M} P_{R}^{\oplus}\right)\right\} \\
& +\min \left\{\frac{1}{2} C\left(\gamma_{M} P_{B}^{\oplus}\right), \frac{1}{2} C\left(\gamma_{M} P_{R}^{\oplus}\right)\right\}=3 R_{M}^{\oplus} \\
\geq & \min \left\{\frac{1}{2} C\left(\gamma_{M} P_{A}^{\dagger}\right), \frac{1}{2} C\left(\gamma_{M} P_{R 1}^{\dagger}\right)\right\} \\
& +\min \left\{\frac{1}{2} C\left(\gamma_{M} P_{B}^{\dagger}\right), \frac{1}{2} C\left(\gamma_{M} P_{R 2}^{\dagger}\right)\right\}=4 R_{M}^{\dagger} .
\end{aligned}
$$

That is,

$$
2 R_{M}^{\star} \geq 3 R_{M}^{\oplus} \geq 4 R_{M}^{\dagger}
$$


On the other hand, with $P_{A}^{\dagger}=P_{A}^{\oplus}=P_{A}^{\star}$ and $P_{B}^{\dagger}=P_{B}^{\oplus}=$ $P_{B}^{\star}$, we can derive

$$
\begin{aligned}
4 R_{E}^{\dagger} & \geq \log \left(1+\gamma_{A E} P_{A}^{\dagger}+\gamma_{B E} P_{B}^{\dagger}+\gamma_{R E}\left(P_{R 1}^{\dagger}+P_{R 2}^{\dagger}\right)\right) \\
& \geq \log \left(1+\gamma_{A E} P_{A}^{\star}+\gamma_{B E} P_{B}^{\star}\right)=2 R_{E}^{\star}
\end{aligned}
$$

and

$$
\begin{aligned}
3 R_{E}^{\oplus} & \geq \log \left(1+\gamma_{A E} P_{A}^{\oplus}+\gamma_{B E} P_{B}^{\oplus}+\gamma_{R E} P_{R 2}^{\oplus}\right) \\
& \geq \log \left(1+\gamma_{A E} P_{A}^{\star}+\gamma_{B E} P_{B}^{\star}\right)=2 R_{E}^{\star} .
\end{aligned}
$$

Therefore, combining (17), (19), (20), and (21), we obtain

$$
\begin{aligned}
& 2 R_{s}^{\star} \geq 2\left[R_{M}^{\star}-R_{E}^{\star}\right]^{+} \geq 4\left[R_{M}^{\dagger}-R_{E}^{\dagger}\right]^{+}=4 R_{s}^{\dagger} \\
& 2 R_{s}^{\star} \geq 2\left[R_{M}^{\star}-R_{E}^{\star}\right]^{+} \geq 3\left[R_{M}^{\oplus}-R_{E}^{\oplus}\right]^{+}=3 R_{s}^{\oplus} .
\end{aligned}
$$

If the power allocation further satisfies $P_{R 1}^{\dagger}=P_{R 2}^{\dagger}$, it is easily established from (3), (4), (8), and (9) that $4 R_{E}^{\dagger}=3 R_{E}^{\oplus}$. Thus, we have $4 R_{E}^{\dagger}=3 R_{E}^{\oplus} \geq 2 R_{E}^{\star}$ from (21), which leads to $2 R_{s}^{\star} \geq 3 R_{s}^{\oplus} \geq 4 R_{s}^{\dagger}$.

\section{B. Optimal Power Allocation}

The optimal power allocation for maximum secrecy rates is to solve (for $k \in\{\dagger, \oplus, \star\}$ )

$$
\begin{array}{ll}
\text { (P.1) } \max _{\mathcal{P}^{k}} & R_{s}^{k} \\
\text { s.t. } & \sum_{p \in \mathcal{P}^{k}} p \leq P_{t} .
\end{array}
$$

Denote the optimal solution to problem (P.1) as $\tilde{R}_{s}^{k}$ and the corresponding set of optimal powers as $\tilde{\mathcal{P}}^{k}$.

Theorem 3. With optimal power allocation, we have $\tilde{R}_{s}^{\star} \geq$ $\frac{3}{2} \tilde{R}_{s}^{\oplus}$ and $\tilde{R}_{s}^{\star} \geq 2 \tilde{R}_{s}^{\dagger}$ at high SNR, i.e., $\gamma_{M}\left(\tilde{P}_{A}^{\star}+\tilde{P}_{B}^{\star}\right) \gg 1$. If the optimal powers $\tilde{P}_{R 1}^{\dagger}$ and $\tilde{P}_{R 2}^{\dagger}$ satisfy $\tilde{P}_{R 1}^{\dagger}=\tilde{P}_{R 2}^{\dagger}$, we have $\tilde{R}_{s}^{\star} \geq \frac{3}{2} \tilde{R}_{s}^{\oplus} \geq 2 \tilde{R}_{s}^{\dagger}$.

Proof: To show $\tilde{R}_{s}^{\star} \geq \frac{3}{2} \tilde{R}_{s}^{\oplus}$ and $\tilde{R}_{s}^{\star} \geq 2 \tilde{R}_{s}^{\dagger}$, we instead show that there exist feasible solutions $R_{s 1}^{\star}$ and $R_{s 2}^{\star}$ such that $R_{s 1}^{\star} \geq \frac{3}{2} \tilde{R}_{s}^{\oplus}$ and $R_{s 2}^{\star} \geq 2 \tilde{R}_{s}^{\dagger}$. Let the sets of optimal powers corresponding to the optimal solutions $\tilde{R}_{s}^{\oplus}$ and $\tilde{R}_{s}^{\dagger}$ be $\tilde{\mathcal{P}}^{\oplus}=$ $\left\{\tilde{P}_{A}^{\oplus}, \tilde{P}_{B}^{\oplus}, \tilde{P}_{R}^{\oplus}\right\}$ and $\tilde{\mathcal{P}}^{\dagger}=\left\{\tilde{P}_{A}^{\dagger}, \tilde{P}_{B}^{\dagger}, \tilde{P}_{R 1}^{\dagger}, \tilde{P}_{R 2}^{\dagger}\right\}$, respectively. Note that $\mathcal{P}_{1}^{\star} \triangleq \tilde{\mathcal{P}}^{\oplus}$ and $\mathcal{P}_{2}^{\star} \triangleq\left\{\tilde{P}_{A}^{\dagger}, \tilde{P}_{B}^{\dagger}, \max \left\{\tilde{P}_{R 1}^{\dagger}, \tilde{P}_{R 2}^{\dagger}\right\}\right\}$ are both feasible for Protocol 3 and hence from Theorem 2 the resulting rates would satisfy $R_{s 1}^{\star} \geq \frac{3}{2} \tilde{R}_{s}^{\oplus}$ and $R_{s 2}^{\star} \geq 2 \tilde{R}_{s}^{\dagger}$, respectively, at high SNR. Moreover, if $\tilde{P}_{R 1}^{\dagger}=\tilde{P}_{R 2}^{\dagger}$, from Theorem 2, we can find a feasible solution $R_{s 1}^{\oplus}$ with the set of powers $\mathcal{P}_{1}^{\oplus}=\left\{\tilde{P}_{A}^{\dagger}, \tilde{P}_{B}^{\dagger}, \max \left\{\tilde{P}_{R 1}^{\dagger}, \tilde{P}_{R 2}^{\dagger}\right\}\right\}$ satisfying (P.1b) to yield $\frac{3}{2} R_{s 1}^{\oplus} \geq 2 \tilde{R}_{s}^{\dagger}$.

\section{Simulation Results}

We model the AWGN channel with channel gain $h_{i j}=$ $\rho_{0} d_{i j}^{-c / 2} e^{i \theta}$, where $d_{i j}$ is the distance between nodes $i$ and $j$, $\rho_{0}$ is a constant, $c$ is the path loss exponent, and $\theta \in[0,2 \pi)$ is the phase shift. We set $\rho_{0}=1, c=3.5$, and the noise power $\sigma_{j}^{2}=-30 \mathrm{dBm}, \forall j$ in our simulation.

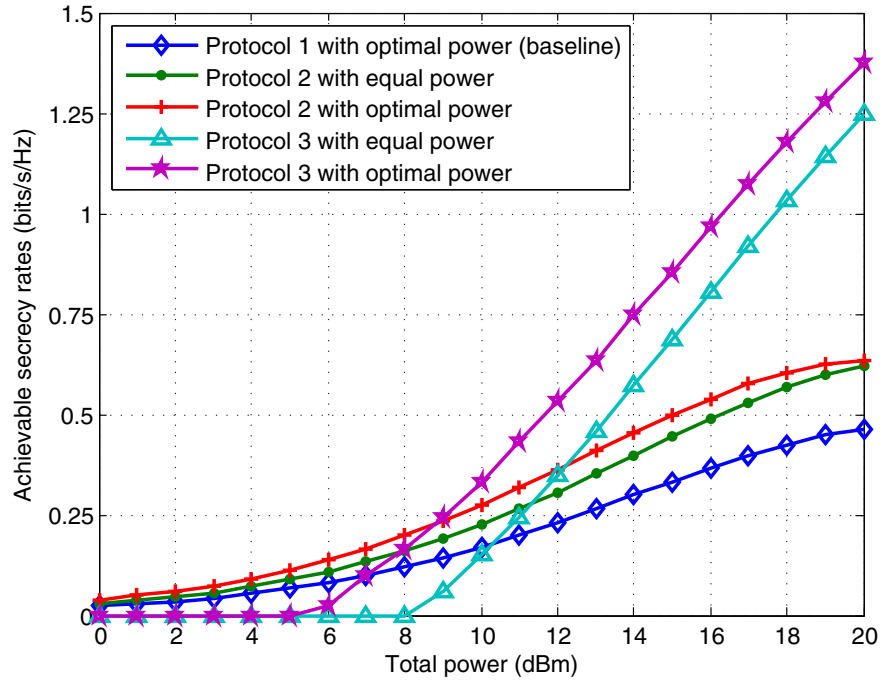

Fig. 2. Achievable secrecy rates vs. total power in a symmetric topology. Equal power allocations for Protocols 2 and 3 are defined in reference to the optimal power allocation for Protocol 1.

\section{A. Symmetric Topologies}

First, we consider a symmetric topology where the two users have equal distances to the relay as well as to the eavesdropper (i.e., $\gamma_{A R}=\gamma_{R A}=\gamma_{B R}=\gamma_{R B}=\gamma_{M}$ and $\gamma_{A E}=\gamma_{B E}$ ), and the users are closer to the relay than to the eavesdropper (i.e., $\gamma_{M} / \gamma_{B E} \simeq 32$ ). Due to topological symmetry, the optimal power allocation yields $\tilde{P}_{A}^{k}=\tilde{P}_{B}^{k}$ for $k \in\{\dagger, \oplus, \star\}$ and $\tilde{P}_{R 1}^{\dagger}=\tilde{P}_{R 2}^{\dagger}$. The optimal powers can be obtained by solving problem (P.1) using the Lagrange method. The optimal powers for Protocol 1, which can be easily established as $P_{M} \triangleq \tilde{P}_{R 1}^{\dagger}=\tilde{P}_{R 2}^{\dagger}=\tilde{P}_{A}^{\dagger}=\tilde{P}_{B}^{\dagger}$, is adopted as a reference point (baseline) for equal power allocation for Protocols 2 and 3 (e.g., the equal power allocation for Protocol 2 is $P_{A}^{\oplus}=$ $\left.\tilde{P}_{A}^{\dagger}=P_{M}, P_{B}^{\oplus}=\tilde{P}_{B}^{\dagger}=P_{M}, P_{R}^{\oplus}=\max \left\{\tilde{P}_{R 1}^{\dagger}, \tilde{P}_{R 2}^{\dagger}\right\}=P_{M}\right)$. Fig. 2 plots the achievable secrecy rates vs. the total power constraint $P_{t}$. Protocol 3 outperforms Protocols 1 and 2 in both power allocation scenarios at high SNR. When $P_{t}=100 \mathrm{~mW}$ $(20 \mathrm{dBm})$, where $\gamma_{M}\left(P_{A}^{\star}+P_{B}^{\star}\right) \gg 1$ and $\gamma_{M}\left(\tilde{P}_{A}^{\star}+\tilde{P}_{B}^{\star}\right) \gg 1$ are satisfied, we observe:

- For equal power allocation, Protocol 3 outperforms Protocol 2 by $101 \%$ and Protocol 2 outperforms Protocol 1 by $34 \%$, i.e., $R_{s}^{\star} \simeq 2.01 R_{s}^{\oplus} \simeq 2.68 R_{s}^{\dagger}$. This verifies $R_{s}^{\star} \geq \frac{3}{2} R_{s}^{\oplus} \geq 2 R_{s}^{\dagger}$ established in Theorem 2 .

- For optimal power allocation, Protocol 3 outperforms Protocol 2 by $116 \%$ and Protocol 2 outperforms Protocol 1 by $37 \%$, i.e., $\tilde{R}_{s}^{\star} \simeq 2.16 \tilde{R}_{s}^{\oplus} \simeq 2.95 \tilde{R}_{s}^{\dagger}$. This verifies $\tilde{R}_{s}^{\star} \geq \frac{3}{2} \tilde{R}_{s}^{\oplus} \geq 2 \tilde{R}_{s}^{\dagger}$ established in Theorem 3.

Fig. 3 plots the achievable secrecy rates vs. $\gamma_{M} / \gamma_{B E}$ in a symmetric topology. We fix the locations of nodes $A$, $B$, and $R$, and increase the distance $d_{B E}$ such that $\gamma_{B E}$ decreases with fixed $\gamma_{M}$. When the eavesdropper is located nearer to the relay and the two users (i.e., $\gamma_{M} / \gamma_{B E} \simeq 1$ ), the eavesdropper has almost the same decoding ability as the 


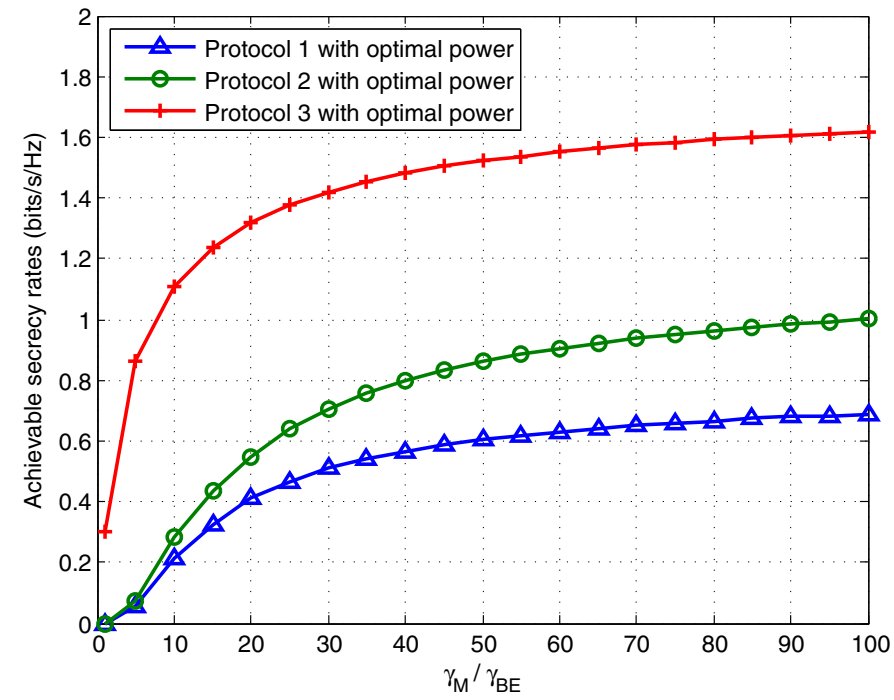

Fig. 3. Achievable secrecy rates vs. $\gamma_{M} / \gamma_{B E}$ in a symmetric topology, where $P_{t}=100 \mathrm{~mW}(20 \mathrm{dBm})$. The eavesdropper is located increasingly distant from the relay and the two users as $\gamma_{M} / \gamma_{B E}$ increases.

relay, and since the relay needs to decode both codewords $X_{A}^{\dagger}$ and $X_{B}^{\dagger}$ (or $X_{A}^{\oplus}$ and $X_{B}^{\oplus}$ ) in Protocol 1 (or Protocol 2), the same codewords are also accessible at the eavesdropper. Thus, no secrecy is achieved, i.e., $\tilde{R}_{s}^{\dagger}=\tilde{R}_{s}^{\oplus} \simeq 0$. In contrast, since the eavesdropper can access only the combined codeword $X_{R}^{\star}$ rather than individual codewords in Protocol 3, the two-way relay system achieves a positive secrecy capacity $\left(\tilde{R}_{s}^{\star}>0\right)$ even when the eavesdropper has the same decoding ability as the relay. The achievable secrecy rate eventually saturates at the two-way relay data rate when the eavesdropper is located increasingly distant from the relay and the two users (large value of $\gamma_{M} / \gamma_{B E}$ ), in which case the eavesdropper has a diminishing impact on the security of the system.

\section{B. Asymmetric Topologies}

Next, we consider an asymmetric topology where the two users have equal distances to the relay but unequal distances to the eavesdropper (i.e., $\gamma_{A E} / \gamma_{B E} \geq 1$ with fixed $\gamma_{B E}$ ). We set $\gamma_{M} / \gamma_{B E} \simeq 32$. Fig. 4 plots the achievable secrecy rates vs. $\gamma_{A E} / \gamma_{B E}$ when $P_{t}=100 \mathrm{~mW}(20 \mathrm{dBm})$. As can be seen, for all the three protocols with optimal power allocation, the achievable secrecy rates are nonincreasing as $\gamma_{A E} / \gamma_{B E}$ increases (i.e., when the topology becomes increasingly asymmetric with respect to the eavesdropper). For Protocol 1 , the achievable secrecy rate decreases negligibly when $\gamma_{A E} / \gamma_{B E} \geq 8$, as in this regime the optimal power allocation will produce $\tilde{P}_{A}^{\dagger} \ll \tilde{P}_{B}^{\dagger}$ and $\tilde{P}_{R 1}^{\dagger} \ll \tilde{P}_{R 2}^{\dagger}$ to avoid sending message $W_{A}$ from user $A$ for maximum secrecy rates, and therefore the secrecy rates become almost independent of $\gamma_{A E} / \gamma_{B E}$. Likewise for Protocol 2, as $\gamma_{A E} / \gamma_{B E}$ increases, the optimal power allocation will produce small $\tilde{P}_{A}^{\oplus}$, and possibly small $\tilde{P}_{A}^{\oplus}$ and $\tilde{P}_{R}^{\oplus}$. However, a small $\tilde{P}_{A}^{\oplus}$ will still allow the eavesdropper to obtain some information about $W_{A}$ by decoding the combined message $X_{A B}^{\oplus}$ from $\left\{Y_{E B}^{\oplus}, Y_{E R}^{\oplus}\right\}$,

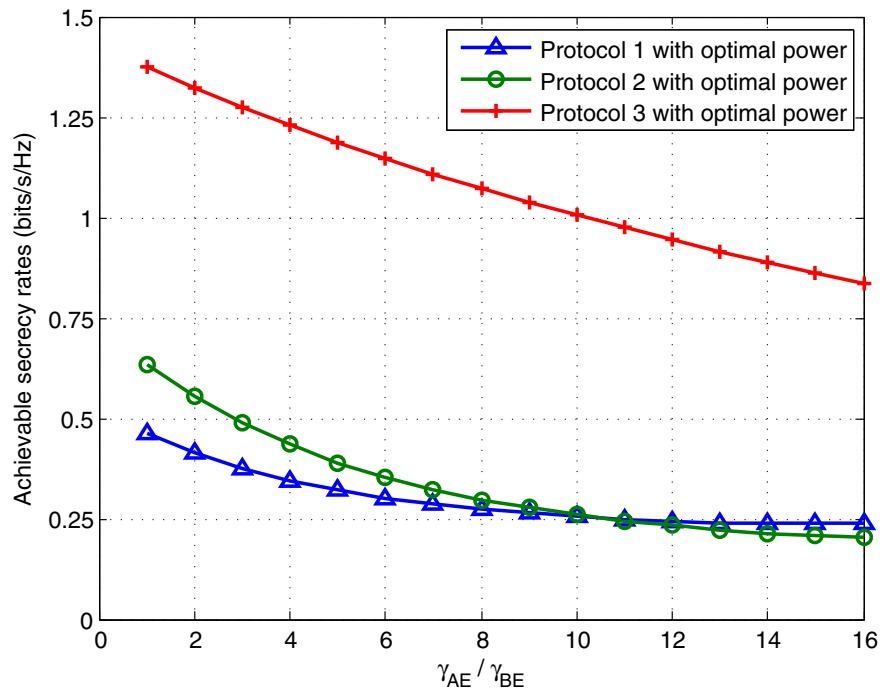

Fig. 4. Achievable secrecy rates vs. $\gamma_{A E} / \gamma_{B E}$ in an asymmetric topology, where $P_{t}=100 \mathrm{~mW}(20 \mathrm{dBm})$.

and small $\tilde{P}_{A}^{\oplus}$ and $\tilde{P}_{R}^{\oplus}$ will compromise the eavesdropper as well as the legitimate receivers. Thus, Protocol 2 might achieve a lower secrecy rate as compared to Protocol 1 in this scenario. Indeed, as shown in Fig. 4, a crossing point exists at around $\gamma_{A E} / \gamma_{B E} \simeq 12$. This is also in accordance with Theorem 3 which suggests that a higher secrecy rate for Protocol 2 is not guaranteed as compared to Protocol 1 when $\tilde{P}_{R 1}^{\dagger}=\tilde{P}_{R 2}^{\dagger}$ is not satisfied, as in an asymmetric scenario.

To examine how this crossing point shifts according to the topology, we plot in Fig. 5 the indifference curve of achievable secrecy rates that represents $\tilde{R}_{s}^{\oplus}=\tilde{R}_{s}^{\dagger}$ in different asymmetric topologies with respect to the eavesdropper. The region below the curve corresponds to $\tilde{R}_{s}^{\oplus}<\tilde{R}_{s}^{\dagger}$, and the region above the curve corresponds to $\tilde{R}_{s}^{\oplus}>\tilde{R}_{s}^{\dagger}$. Three exemplary topologies that correspond to three points on the curve are also shown, where topology $(b)$ represents the case shown in Fig. 4 with the crossing point at $\gamma_{A E} / \gamma_{B E} \simeq 12$. As can be seen from this figure, when the eavesdropper is located near to the relay and the two users (i.e., small $\gamma_{M} / \gamma_{B E}$ ), a slight degree of asymmetry (i.e., small $\gamma_{A E} / \gamma_{B E}$ ) could reverse the relationship of $\tilde{R}_{s}^{\oplus}$ and $\tilde{R}_{s}^{\dagger}$. When the eavesdropper is located distant from the relay and the two users (i.e., large $\left.\gamma_{M} / \gamma_{B E}\right), \tilde{R}_{s}^{\oplus}<\tilde{R}_{s}^{\dagger}$ occurs only when there is a large degree of asymmetry with respect to the eavesdropper (i.e., large $\left.\gamma_{A E} / \gamma_{B E}\right)$.

\section{CONCLUSION}

We have conducted a comparative study of three common two-way relay protocols from the previously unexamined perspective of information-theoretic security. We observed that previous throughput comparison results do not directly generalize to the case with security considerations. Specifically, we showed that CF based on PNC (Protocol 3) achieves the highest secrecy rate at high SNR, while, interestingly, conventional DF (Protocol 1) might outperform the supposedly 

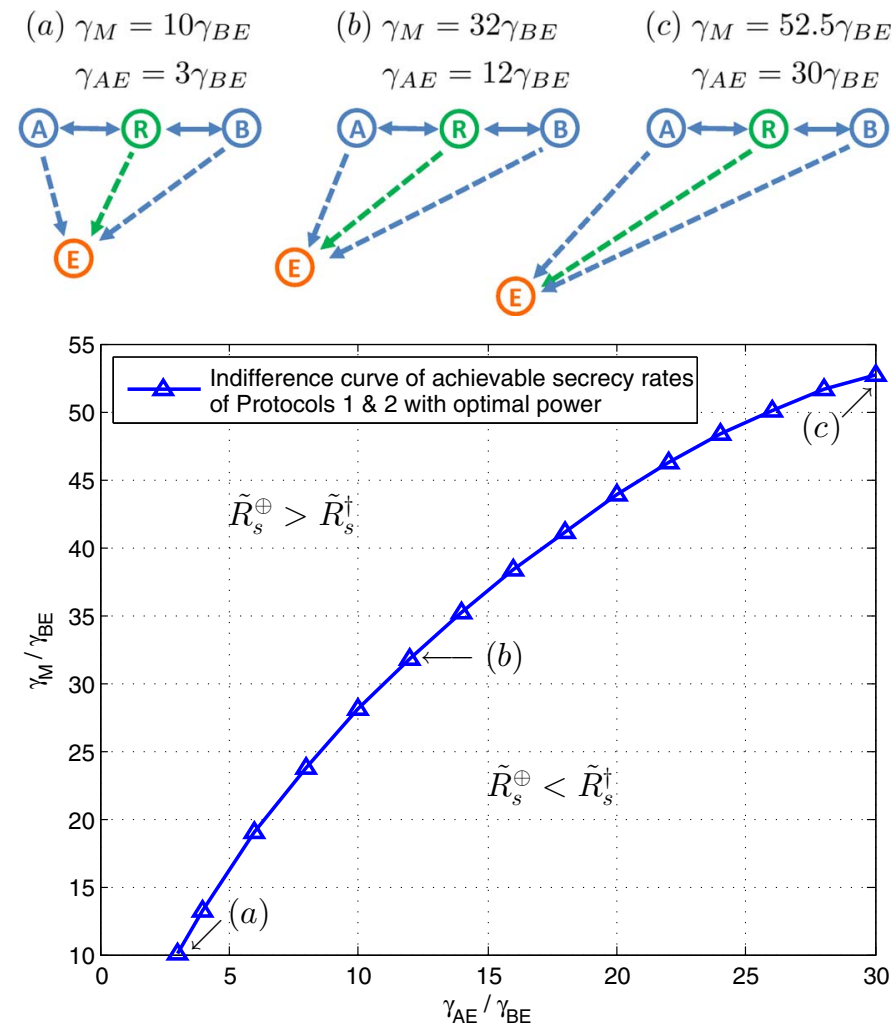

Fig. 5. Indifference curve of achievable secrecy rates of Protocols 1 and 2 in asymmetric topologies, where $P_{t}=100 \mathrm{~mW}(20 \mathrm{dBm})$. Exemplary topologies corresponding to three points on the curve are shown.

enhanced scheme of DF with NC (Protocol 2) in terms of secrecy rates in asymmetric topologies. The crossing points of conventional DF scheme and DF with $\mathrm{NC}$ scheme are also determined in terms of network topologies based on the indifference curve of achievable secrecy rates in a two-way relay system.

\section{REFERENCES}

[1] Y.-S. Shiu, S. Y. Chang, H.-C. Wu, S. C.-H. Huang, and H.-H. Chen, "Physical layer security in wireless networks: a tutorial," IEEE Trans.
Wireless Commun., vol. 18, no. 2, pp. 66-74, Apr. 2011.

[2] C. E. Shannon, "Communication theory of secrecy systems," Bell Syst. Tech. J., vol. 28, pp. 656-715, 1949.

[3] A. D. Wyner, "The wire-tap channel," Bell Syst. Tech. J., vol. 54, no. 8 , pp. 1355-1387, Jan. 1975.

[4] I. Csiszar and J. Korner, "Broadcast channels with confidential messages," IEEE Trans. Inf. Theory, vol. 24, no. 3, pp. 339-348, May 1978.

[5] S. Leung-Yan-Cheong and M. E. Hellman, "The Gaussian wire-tap channel," IEEE Trans. Inf. Theory, vol. 24, no. 4, pp. 451-456, Jul. 1978.

[6] Y. Liang and H. V. Poor, "Multiple-access channels with confidential messages," IEEE Trans. Inf. Theory, vol. 54, no. 3, pp. 976-1002, Mar. 2008.

[7] L. Lai and H. El Gamal, "The relay-eavesdropper channel: Cooperation for secrecy," IEEE Trans. Inf. Theory, vol. 54, no. 9, pp. 4005-4019, Sep. 2008.

[8] E. Ekrem and S. Ulukus, "Secrecy in cooperative relay broadcast channels," IEEE Trans. Inf. Theory, vol. 57, no. 1, pp. 137-155, Jan. 2011.

[9] X. He and A. Yener, "Strong secrecy and reliable Byzantine detection in the presence of an untrusted relay," IEEE Trans. Inf. Theory, vol. 59, no. 1, pp. 177-192, Jan. 2013.

[10] J. Richter, E. Franz, S. Engelmann, S. Pfennig, and E. Jorswieck, "Physical layer security vs. network layer secrecy: Who wins on the untrusted two-way relay channel?" in Proc. IEEE Computer Aided Modeling and Design of Communication Links and Networks (CAMAD) Workshop, Sep. 2013, pp. 164-168.

[11] S. Kim, W.-Y. Shin, and K. Ishibashi, "The Gaussian two-way relay channel with wiretapper," in Proc. Asilomar, Nov 2011, pp. 1987-1991.

[12] H.-M. Wang, Q. Yin, and X.-G. Xia, "Distributed beamforming for physical-layer security of two-way relay networks," IEEE Trans. Signal Processing, vol. 60, no. 7, pp. 3532-3545, July 2012.

[13] A. Mukherjee and A. Swindlehurst, "Securing multi-antenna two-way relay channels with analog network coding against eavesdroppers," in Proc. IEEE SPAWC, June 2010, pp. 1-5.

[14] Y. Wu, P. A. Chou, and S. Y. Kung, "Information exchange in wireless networks with network coding and physical-layer broadcast," Microsoft Research Technical Report MSR-TR-2004-78, Aug. 2004.

[15] S. Zhang, S. C. Liew, and P. P. Lam, "Hot topic: Physical-layer network coding," in Proc. ACM MobiCom, Los Angeles, CA, Sep. 2006, pp. $358-365$.

[16] M. P. Wilson, K. Narayanan, H. D. Pfister, and A. Sprintson, "Joint physical layer coding and network coding for bidirectional relaying," IEEE Trans. Inf. Theory, vol. 56, no. 11, pp. 5641-5654, Nov. 2010.

[17] P. Popovski and H. Yomo, "The anti-packets can increase the achievable throughput of a wireless multi-hop network," in Proc. IEEE ICC, Istanbul, Turkey, Jun. 2006, pp. 3885-3890.

[18] B. Nazer and M. Gastpar, "Reliable physical layer network coding," Proc. IEEE, vol. 99, no. 3, pp. 438-460, Mar. 2011.

[19] J. Chen, L. Song, Z. Han, and B. Jiao, "Joint relay and jammer selection for secure decode-and-forward two-way relay communications," in Proc. IEEE GLOBECOM, Dec 2011, pp. 1-5. 